\title{
A Study on Priority Based ZigBee Network Performance Analysis with Tree Routing Method
}

\author{
Nazrul Islam, Md. Jaminul Haque Biddut, Asma Islam Swapna, Mehedy Hasan Rafsan Jany \\ Department of Information and Communication Technology (ICT), Mawlana Bhashani Science and \\ Technology University, Santosh, Tangail-1902, Bangladesh \\ Email: nazrul.islam@mbstu.ac.bd, biddutict@gmail.com, asma0swapna@gmail.com, rafsanjany@outlook.com
}

Received 1 July 2015; accepted 28 July 2015; published 31 July 2015

Copyright (C) 2015 by authors and Scientific Research Publishing Inc.

This work is licensed under the Creative Commons Attribution International License (CC BY). http://creativecommons.org/licenses/by/4.0/

(c) (i) Open Access

\begin{abstract}
The Wireless Sensor Network (WSN) is spatially distributed autonomous sensor to sense special task. WSN like ZigBee network forms simple interconnecting, low power, and low processing capability wireless devices. The ZigBee devices facilitate numerous applications such as pervasive computing, security monitoring and control. ZigBee end devices collect sensing data and send them to ZigBee Coordinator. The Coordinator processes end device requests. The effect of a large number of random unsynchronized requests may degrade the overall network performance. An effective technique is particularly needed for synchronizing available node's request processing to design a reliable ZigBee network. In this paper, region based priority mechanism is implemented to synchronize request with Tree Routing Method. Riverbed is used to simulate and analyze overall ZigBee network performance. The results show that the performance of the overall priority based ZigBee network model is better than without a priority based model. This research paves the way for further designing and modeling a large scale ZigBee network.
\end{abstract}

\section{Keywords}

WSN, ZigBee Network, Tree Routing Method, Performance Analysis, Riverbed

\section{Introduction}

A Wireless Sensor Network (WSN) is a collection of spatially distributed sensor devices or nodes working together. WSN is a special type ad-hoc network which can collect data from the surrounds, process the data and then transmit to other devices or the sink. It has widespread use in monitoring different environment and physi-

How to cite this paper: Islam, N., Biddut, Md.J.H., Swapna, A.I. and Jany, M.H.R. (2015) A Study on Priority Based ZigBee Network Performance Analysis with Tree Routing Method. Journal of Computer and Communications, 3, 1-10. 
cal conditions such as temperature, pressure, sound and vibrations, etc. Being advantageous compared to the wired and wireless network, WSN has remarkable limitations in battery capacity, short distance, and networking in small range. Remaining most of the characteristics same, a new WSN standard-ZigBee was introduced having low power dissipation facility for wide range use [1] [2]. ZigBee adopts the specification standard of IEEE 802.15.4 which enables communication and interoperability among simple and smart objects. ZigBee provides the foundation for the Internet of Things.

ZigBee is enlarging the field of Internet of Things rapidly, offering better efficiency in Sensor to Machine and Machine to Machine communication wirelessly. ZigBee is in the area of Internet of Things in industrial monitoring and control system, smart home environment, hospital automation, remote sensing, smoke and heat sensing, smart energy management, connected lighting and so on [2]-[5]. ZigBee Routers, ZigBee end devices and a ZigBee Coordinator implement the ZigBee network [6]. ZigBee Coordinator acts like a cloud and is similar to the cloud of the Internet of Things. ZigBee network topologies such as star, tree, cluster and mesh can support up to 65,000 nodes for a wide range of areas [7]. The ZigBee stacks architecture [8] consists of critical four layers: Physical (PHY) layer, Medium Access Control (MAC) sub-layer, network (NWK) layer and the framework for the application layer. The application layer includes the application support sub-layer (APS), the ZigBee device objects (ZDO) and the manufacturer-defined application objects. ZDO provides key functions for managing and gathering system properties.

The analysis of ZigBee network performance is getting more interesting. Though the ZigBee technology is introduced recently, several studies have been performed on the network performance analysis of the ZigBee network where CSM/CA, routing performance, effective data flow and scheduling have been focused [9] [10]. The study [1] proposed priority based energy balanced ZigBee network. But all these researches fail to define request synchronization in Coordinator among different nodes. There is a gap in forming large scale ZigBee network and to build and manage different regions in the ZigBee network. Also nodes in different regions and how to synchronize their request have not been studied yet. The drawbacks of these researches allow us to design new network architecture for an efficient ZigBee network and then analyze the network performance. The new network architecture assigns priority mechanism to Tree routing and performs better than others.

The goal of this paper is to represent a priority based ZigBee network with Tree routing, which provides better network performance. In this design, region synchronization is constructed on the basis of priority. A child node (end node or router) connects to a parent node. The child nodes pay willingly for the connection. Based on the assigned regional priority in the network, each end device of higher priority has a higher willingness to pay and vice versa.

The structure of this paper is as follows. In Section 2, the research methodology is described. In Section 3, proposed model design and implementation in Riverbed are illustrated. Section 4 evaluates the performance to show that results meet up with designs objectives. Section 5 concludes the paper with future work.

\section{Research Method}

A literature review has been performed to analyze the ZigBee network performance of the proposed priority based regions with Tree routing. In a ZigBee network, region synchronization is practiced based on the assigned priority to different regions in a tree routing. Before this, a careful study is done on how ZigBee network works. A simulation tool was necessary to analyze the performance of the proposed prioritized regions model. Several comparative studies have been performed among NS-2, NS-3, OMNET++, MATLAB, and Riverbed. Studies show that Riverbed has good potential to perform precisely in simulating the ZigBee WSN; contrarily NS-2 has lower real world application efficiency. Riverbed installation and configuration are easier than others. It is user friendly and has a wide range of acceptance. The Riverbed was installed in a core i5 processor based workstation.

The ZigBee protocol supports a vast variety of data for simulation for different layers. Simulated data of different ZigBee layers are collected appropriately. Different graphs are plotted after analyzing the simulated data. The effect of different network parameters reflects on the graphs which offer an analysis of network performance.

\section{Modeling and Simulation}

For modeling the ZigBee network four process was required in Riverbed-ZigBee MAC layer model, ZigBee 
Application layer model, ZigBee network layer model and Carrier Sense Multiple Access/Collision Avoidance model (CSMA/CA) [11]. For Tree topology implementation in Riverbed a number of ZigBee end devices were connected to the coordinators with number of routers for routing packet in the destinations.

In this paper, the main theme is to implement a ZigBee network that bases on priority based region with Tree Routing Method. The Tree routing is simple, reliable and efficient routing algorithm of any variety of destination address. In ZigBee, network addresses are assigned using distributed addressing scheme. Due to such addressing scheme, a Tree topology has constructed for network; each end device can manage the address space of its descendant. If the destination address is in the address space that a node is made out, the node forwards the packet to one of its child nodes. Otherwise, packet is forwarded its parent node.

In the same procedure, the parent or child which receives the data packet selects the next hop address. In the view point of memory usage, Tree routing is more efficient, but sometimes routing cost may inefficient. All the devices in the topology was simulated using Request-to-Send/Clear-to-Send (RTS/CTS) handshaking on delay and other Quality of Service (QoS) parameters [12]. It allows thousands of other nodes to be deployed throughout the network sensor field. Using self-assigning Personal Area network ID (PAN ID) ZigBee topology formed a network. After that the network is configured for accept and decline the parent node requests to the Child. Table 1 contains the network parameters needed for the environment setup and desired simulation.

All the nodes in the working plane were supposed to 1 meter higher above the ground. Depending on the parameters of Table 1, the simulation was to run about a definite period over the MAC, Application and Physical layer parameters described in Table 1. Using those parameters the network traffic was created based on the device to Coordinator configuration in this implementation.

End devices were set up in different ranges which create a problem of region synchronization as they are connected with several routers. Hence, the main task was to find the priority based region for first execution of requests from the parents leaving others behind. The research came with the solution of router-Coordinator distance. This distance is called area factor or priority based region including many end devices and a priority defining factor. However, the priority based region was set here based on the Table 2 region payment module.

Table 1. ZigBee Mac, Physical and Network layer configuration.

\begin{tabular}{ccc}
\hline & MAC Layer Parameters & \\
\hline ACK status & enabled \\
ACK wait duration & 0.05 \\
Number of retransmission & 5 \\
Minimum backoff exponent & 3 \\
Maximum no of backoffs & Physical Layer Parameters & \\
Channel sensitivity duration & & 0.1 \\
\hline Data rate & Auto calculate \\
\hline Packet reception-power threshold & -85 \\
Transmission band & 2450 MHz band \\
Transmission power & Network Layer Parameters & 0.05 \\
\hline Destination & Uniform (20.21) \\
\hline Packet interval time & Infinity \\
Packet size & Coordinator \\
\hline Start time & Constant (1024) \\
\hline
\end{tabular}


Table 2. Used region payment module.

\begin{tabular}{ccc}
\hline Region Name & Unit Price & Available Nodes \\
\hline Region 1 & 700 & Node 1, node 18, node 19, node 20, node 21 \\
Region 2 & 500 & Node 2, node 15, node 16, node 17, node 23 \\
Region 3 & 400 & Node 3, node 5, node 6, node 7, node 24 \\
Region 4 & 600 & Node 4, node 8, node 9, node 10, node 25 \\
Region 5 & 1200 & Node 0, node 11, node 12, node 13, node 14 \\
\hline
\end{tabular}

By these values in Table 2 the importance of the requests from each regions are setup. This gives the assumption of the priority based network illustrated in the Figure 1. The diagram holds four priority based regions for both the requesting periods and execution periods. Here, nodes from two different priority requests for communication at the same time with the Coordinator. When, those requests are received in the router, it checked for priority parameter and executes for response for the highest priority regions, here for $2^{\text {nd }}$ priority region. Other regions in the same color represents that they are out of requests or order at that moment.

After scenario configuration the statistics were selected for result analysis. Simulation was run for a specific period of time. After simulation, region based priority ZigBee network model shows routing layout as depicted in Figure 2 and then results were captured. Table 3 shows the setup values for simulation in this study.

The Riverbed simulation of the proposed ZigBee network model is evaluated for analyzing the various network performance metrics. Application and MAC layer performance parameters include sent and received traffic statistics, end to end delay among the layers, packet delivery ratio, retransmission attempts and throughput over the channels.

\section{Results and Analysis}

This section offers competitive description about the analysis of the obtained discrete event simulation result. The result is based on the ZigBee's various layers performance analysis. The graphs are generated by using different parameters (packet delivery ratio, retransmission attempt, media access delay, queue size and queuing delay, data dropped, hop number etc.).

\subsection{Packet Delivery Ratio}

Packet Delivery Ratio (PDR) is adopted as one of the main performance metric. Average Packet delivery ratio is used for all the end nodes. It can be calculated as the ratio in between the total number of data packets that are received by the sink and the total number of data packets that are sent by all end nodes. The ratio is between the total number of data packets that are received by the coordinator (node_0) and the total number of data packets that are sent by all end nodes.

Figure 3 illustrates for without priority scenario, PDR value is almost 0.85 . In the other word, $85 \%$ of data packets are successfully received by the Coordinator.

Figure 4 illustrates for with priority scenario, PDR value is almost 0.92 . In the other word, $92 \%$ of data packets are successfully received by the Coordinator.

In priority based model, data packets are delivered to destination through queue on the basis of their priority. Collision and other queuing time out packet loss is less than without priority based model. The average packet delivery ratio is always higher in priority based model.

\subsection{Retransmission Attempts}

Total number of retransmission attempts by all 802.15.4 MACs in the network until either packet is successfully transmitted or it is discarded as a result of reaching the retry limit.

Figure 5 is described for both with and without priority scenarios; retransmission attempts are almost same for global network and Coordinator (node_0). 


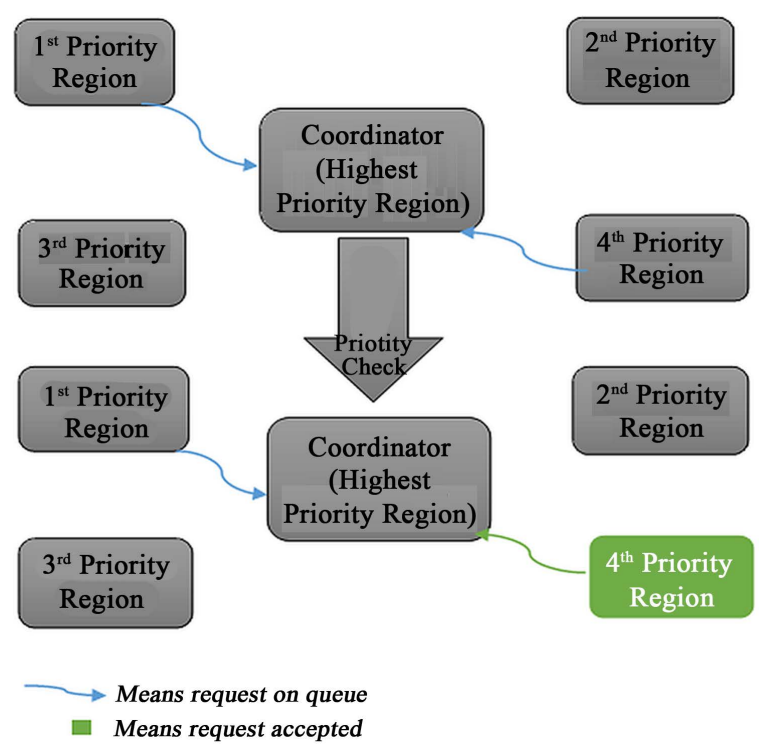

Figure 1. Block diagram of priority based ZigBee Network model.

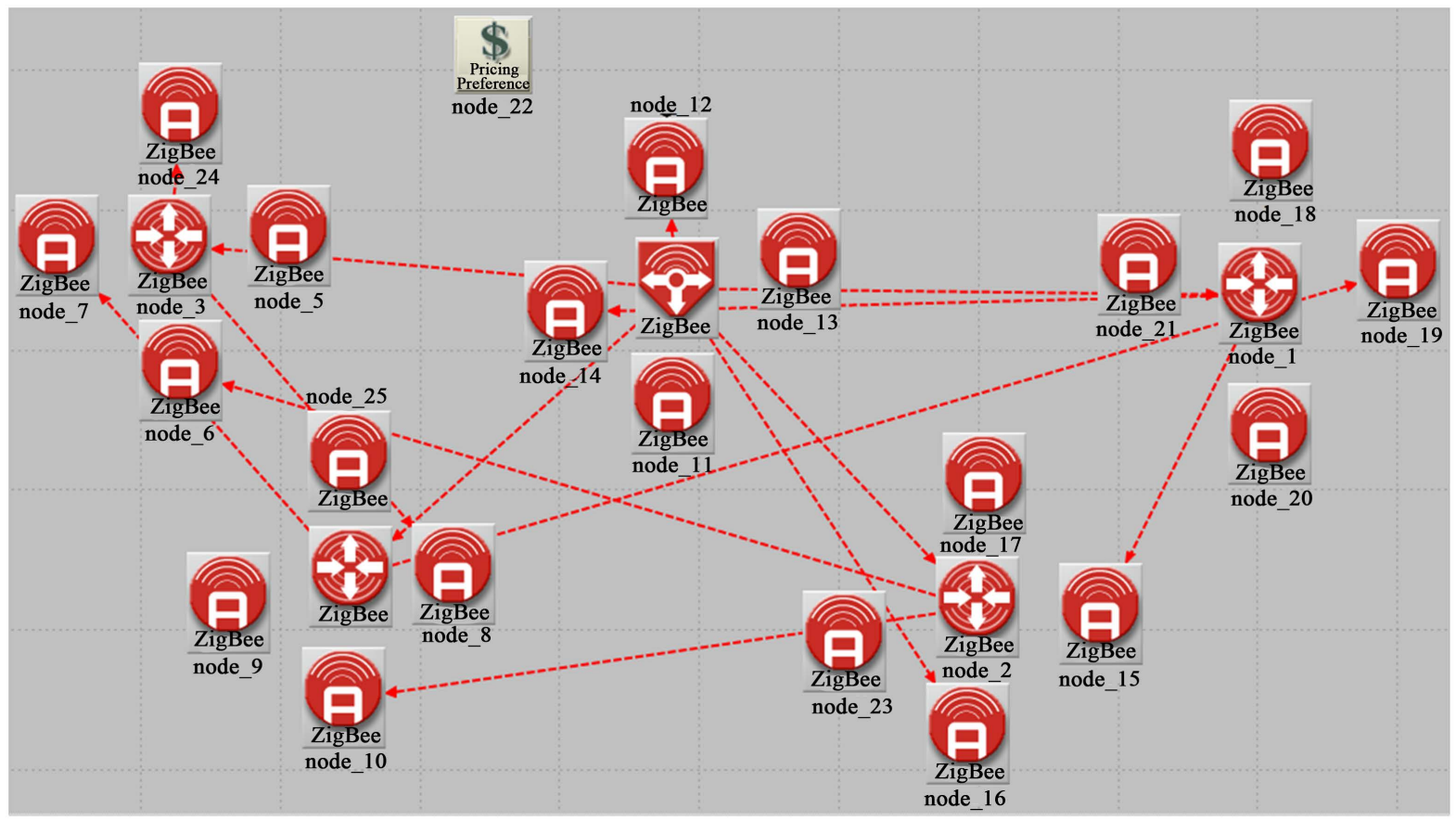

Figure 2. ZigBee network routing layout after simulation.

Table 3. Simulation parameters for ZigBee network model.

\begin{tabular}{ccc}
\hline Parameters & Values \\
\hline Number of nodes & 24 \\
Number of coordinator & 1 \\
Number of router & 4 \\
Number of end devices & $300 \times 300(\mathrm{~m})$ \\
Network dimension & $600 \mathrm{sec}$. \\
\hline
\end{tabular}


Packet Delivery Ratio for Without Priority

—_Region_Based-without_priority_RECOVERED-DES-1: ZigBee Application.Traffic Sent (packets/sec) 〈PAN 0>

_ Region_Based-without_priority_RECOVERED-DES-1: Office Network.node_0.ZigBee Application.Traffic Received (packets/sec)

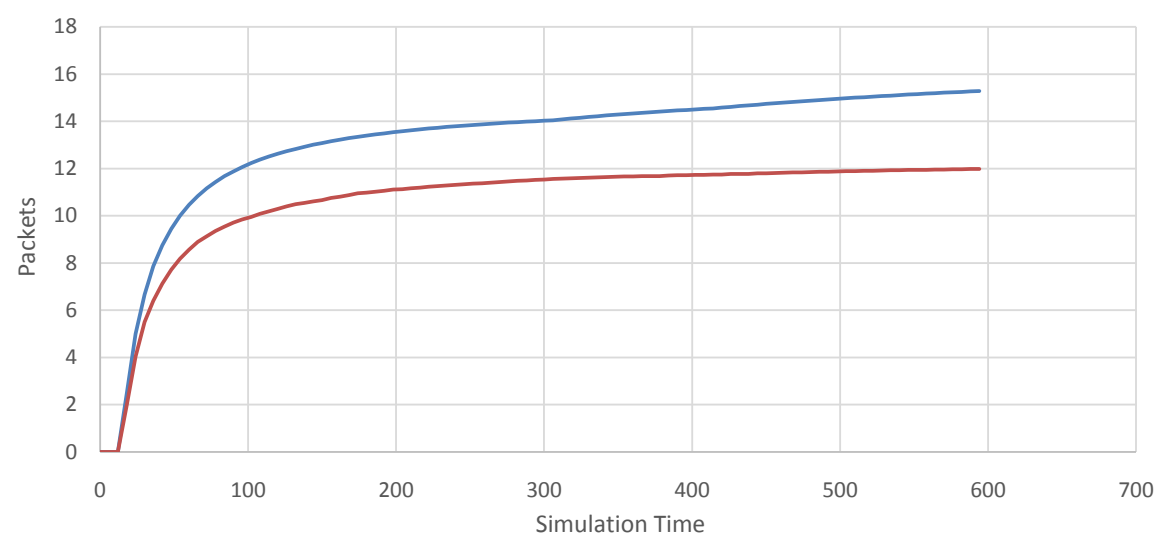

Figure 3. Packet delivery ratio for without priority scenario.

Packet Delivery Ratio for With Priority

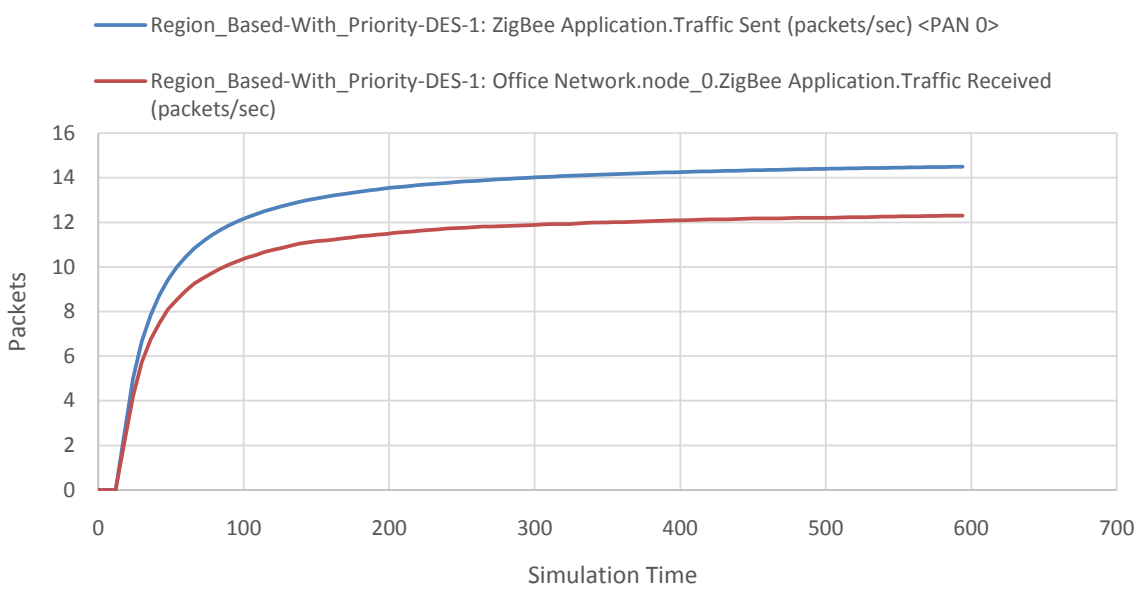

Figure 4. Packet delivery ratio with priority scenario.

Retransmission Attempt

— Region_Based-With_Priority-DES-1: ZigBee 802_15_4 MAC.Retransmission Attempts (packets)

—Region_Based-without_priority_RECOVERED-DES-1: ZigBee 802_15_4 MAC.Retransmission Attempts (packets)

— Region_Based-With_Priority-DES-1: Office Network.node_0.ZigBee 802_15_4 MAC.Retransmission Attempts (packets)

—Region_Based-without_priority_RECOVERED-DES-1: Office Network.node_0.ZigBee 802_15_4 MAC.Retransmission Attempts (packets)

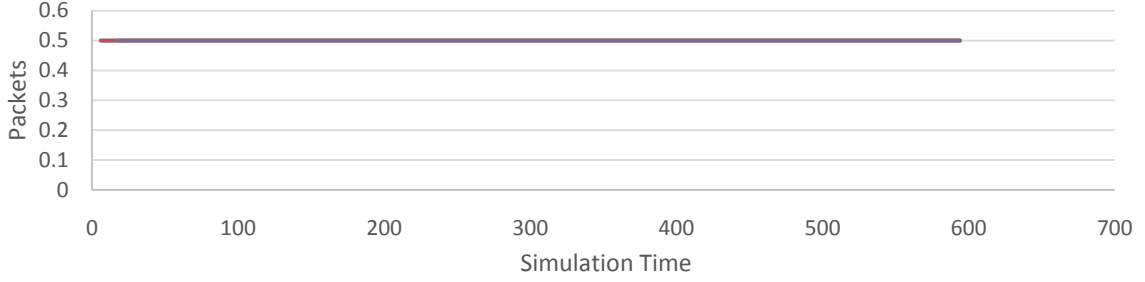

Figure 5. Retransmission attempt (packets) for global network and coordinator (node_0). 


\subsection{Media Access Delay}

The total queuing and contention delays of the data frames transmitted by all the 802.15.4 MAC (Medium Access Control).

For each frame (see Figure 6), this delay is calculated as the duration from a time when it is inserted into the transmission queue, which is arrival time for higher layer data packets and creation time for all other frames types, until the time when the frame is sent to the physical layer for the first time. Media access delay is slightly higher for with priority model than without priority for the global network model. On the other hand, for Coordinator media access delay is lower in with priority model than without priority.

\subsection{Queue Size and Queuing Delay}

Queue size represents total number of packets outstanding in the MAC Queue. That indicates the number of a sequence of request waiting to execute in Queue.

Figure 7 represents the average queue size for the Coordinator in with priority model is smaller than without priority model.

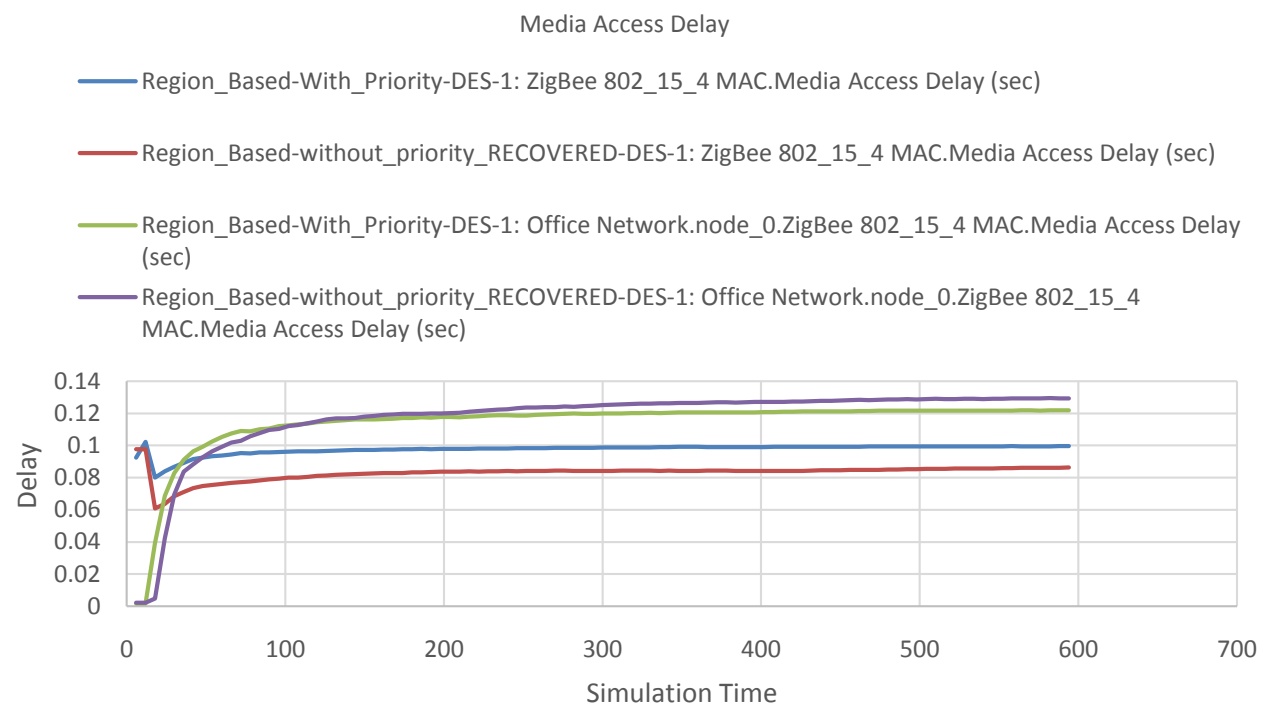

Figure 6. Media access delay (sec) for global network and coordinator (node_0).

Queue Size

—_Region_Based-With_Priority-DES-1: Office Network.node_0.ZigBee 802_15_4 MAC.Queue Size (packets)

—Region_Based-without_priority_RECOVERED-DES-1: Office Network.node_0.ZigBee 802_15_4 MAC.Queue Size (packets)

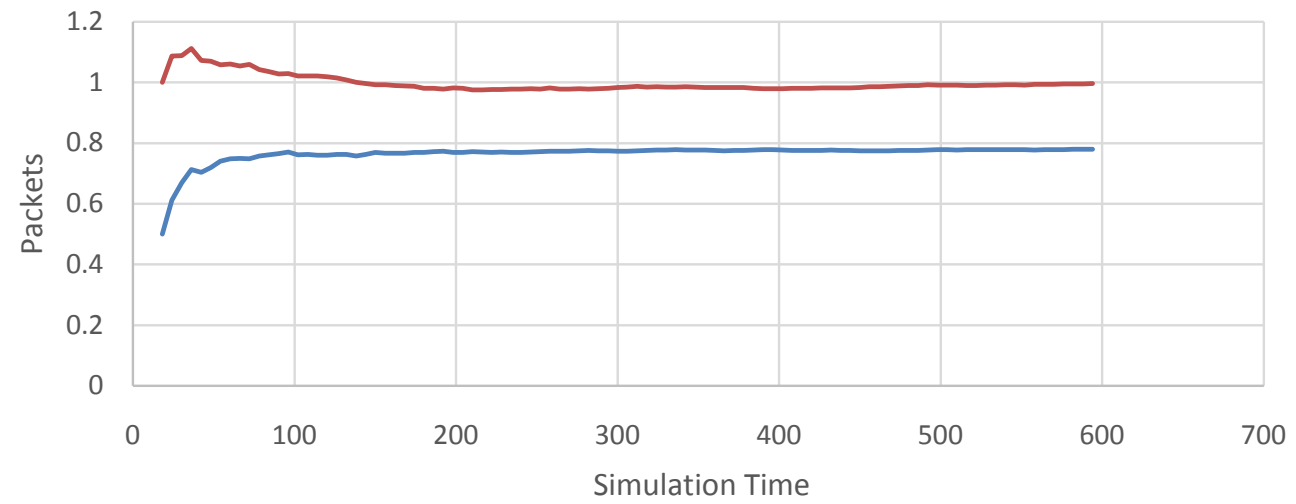

Figure 7. Queue size (packets) for coordinator (node_0). 
Queuing delay represents the queuing delay that packets from the network layer incurs at the MAC queue until it can be executed. It is the main component of overall network delay.

Figure 8 illustrates in without priority model has huge queuing delay for Coordinator (node_0) than with priority model.

The priority model discards packets on the basis of their priority. The only eligible packets are permitted to stay in the queue and for this reason queue size is always smallerand queuing delay also lower for with priority based model.

\subsection{Data Dropped (Retry Threshold Exceeded)}

Higher layer data traffic (in bits/sec) dropped by the 802.15.4 MAC due to consistently failing transmission or retransmissions.

This statistic reports the number of the higher layer packets that are dropped because the MAC could not receive any ACKs for the re-transmissions of those packets or their fragments, and the packets retry counts reached MAC's retry limit or failure to transmit a packet due to channel access failure (reaching maximum backoff limit). This statistic also reports the outstanding packets in the buffers that are dropped during roaming.

Figure 9 describes the data dropped rate for global network and Coordinator (node_0) is always lower in with priority model than without priority model. In without priority based model, data dropped possibility grows for inter packet collision and queuing time out. Moreover, loss of acknowledgements increases retry threshold for sender nodes in without priority based model than with the priority based model.

\subsection{Hop Numbers}

Average number of hops traveled by application traffic in the PAN. It is the number of times a packet travels from the source throughout the intermediate nodes to reach the destination.

Figure 10 shows the average hop numbers of priority based architecture has always higher than without priority based architecture. In this Figure 10, there are two parts. Upper part represents the average number of hops for with priority policy and here the blue horizontal line represents the number of hops for with priority policy. On the other hand, lower part represents the average number of hops for without priority policy and here the blue horizontal line represents the number of hops for without priority policy. The more hop number of a network represents it has more layered architecture than the other.

In this paper, various characteristics of ZigBee network performance has analyzed. The priority mechanism is setup for ZigBee network among various regions. Almost in all the statistics shown that priority based network performance is going prominent than without priority based network.

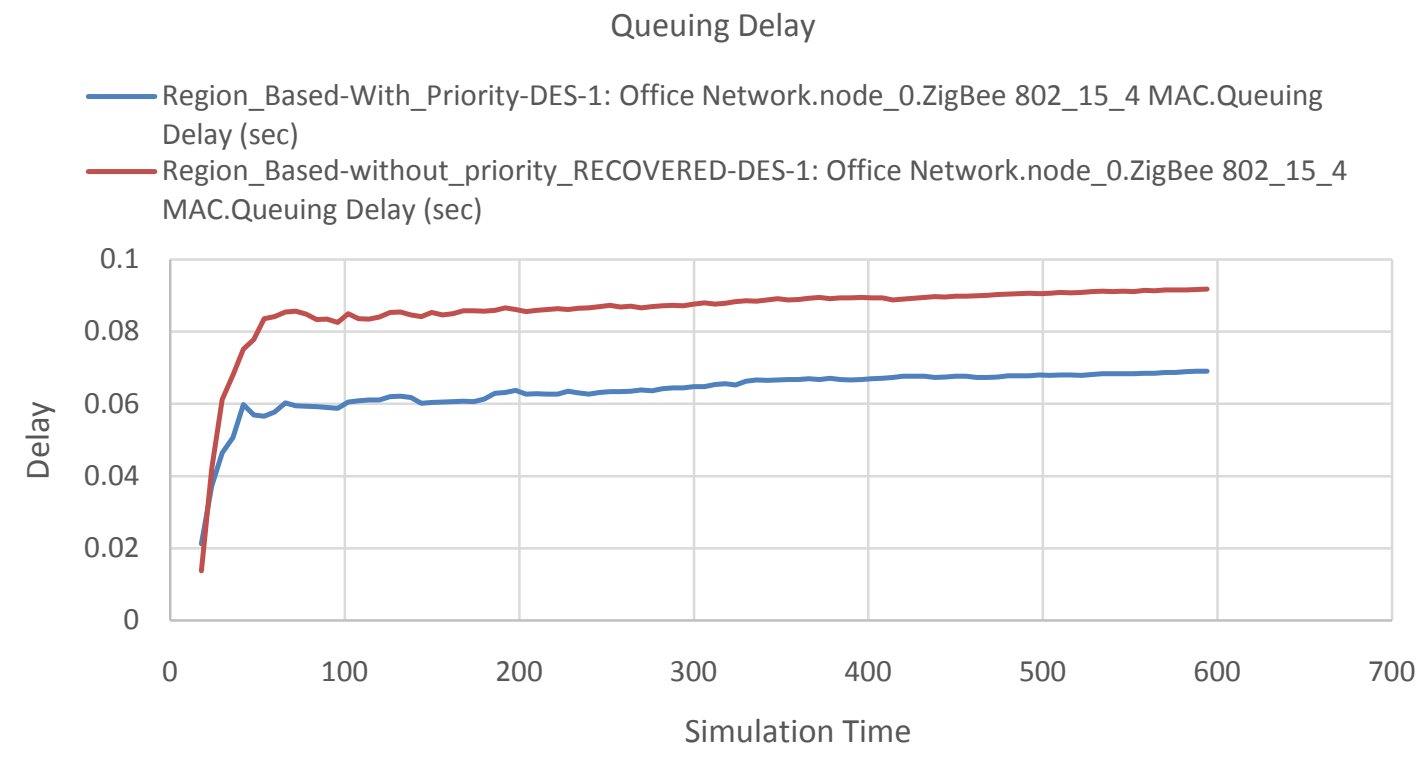

Figure 8. Queuing delay (sec) for coordinator (node_0). 
Data Dropped (retry Threshold)

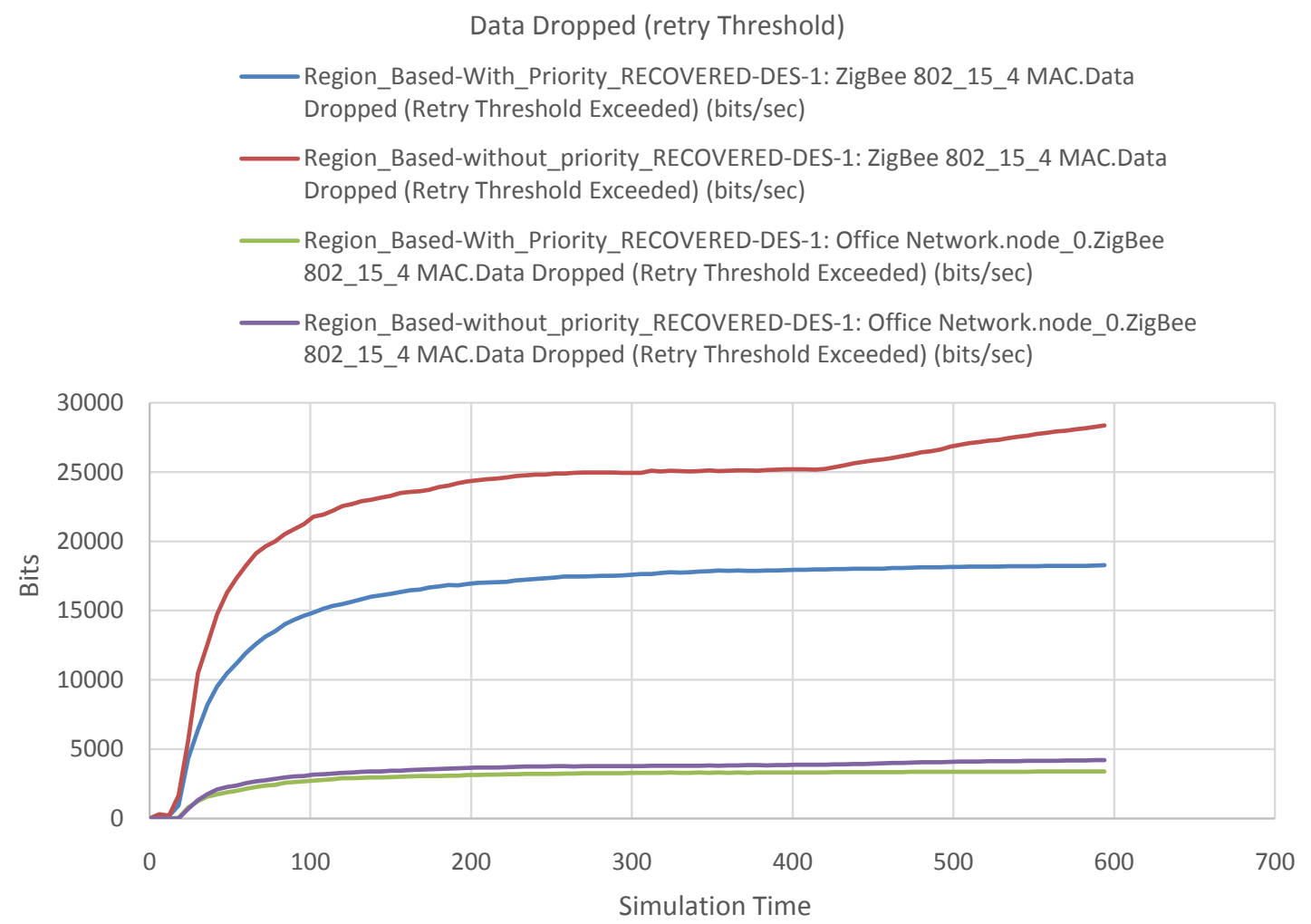

Figure 9. Data dropped (bits/sec) for global network and coordinator (node_0).

Hop Numbers

_ Region_Based-With_Priority-DES-1: ZigBee Network Layer.Number of Hops <PAN 0>

_Region_Based-Without_Priority-DES-1: ZigBee Network Layer.Number of Hops <PAN 0>

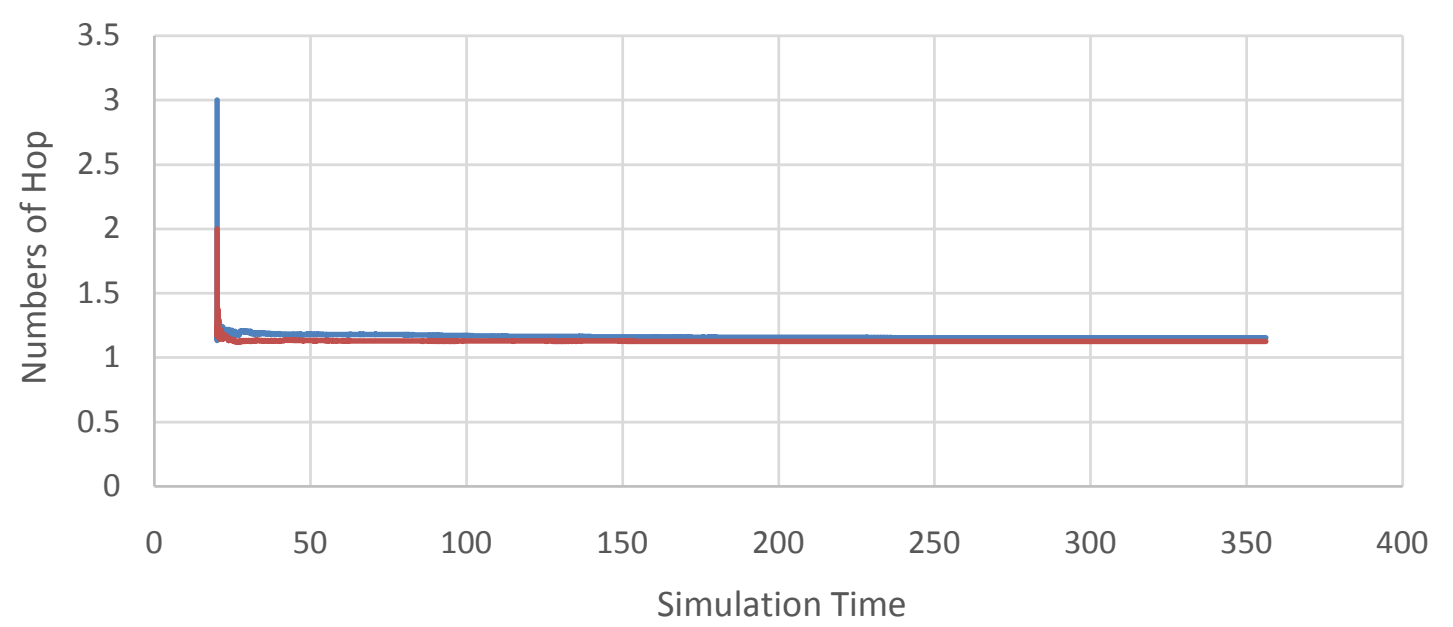

Figure 10. Average hop numbers for with and without priority model.

\section{Conclusions}

This paper proposes region based priority technique to evaluate the overall performance of ZigBee network. The results were attained by performing an extensive simulation study. The result comes to several conclusions on the basis of packet delivery ratio, re-transmission attempts, media access delay, queue size, queuing delay, and 
data dropped threshold and hop number. The outcomes of this study based on performance metrics for priority based model are as follows: First, packet delivery ratio has increased up to $6 \%$ for priority based model and retransmission attempts almost the same for both priority based and no priority based model; Second, smaller queue size, lower queuing delay and media access delay for priority based model; Third, low data drop threshold and more hop numbers for priority based model. This defines that the comprehensive performance of proposed priority based model is more reliable and robust than no priority based model. This study also shows that region based priority approach is more convenient for forming an efficient ZigBee network.

Future work includes load balancing scheme for large scale ZigBee network, effective mobility management for ZigBee mobile sensor nodes and analyzing impact of ZigBee's radio frequency on environment.

\section{References}

[1] Wang, J., Chen, M. and Leung, V.C. (2013) Forming Priority Based and Energy Balanced ZigBee Networks-A Pricing Approach. Telecommunication Systems, 52, 1281-1292.

[2] Modeler (2015) Riverbed Technology.

[3] Baviskar, J., Mulla, A., Upadhye, M., Desai, J. and Bhovad, A. (2015) Performance Analysis of ZigBee Based Real Time Home Automation System. 2015 International Conference on Communication, Information \& Computing Technology (ICCICT), 15-17 January 2015, Mumbai, 1-6. http://dx.doi.org/10.1109/ICCICT.2015.7045685

[4] Zhu, X.Q. and Wang, J.M. (2006) The Research and Implementation of ZigBee Protocol Network. Journal of Electronic Technology, 1, 129-132.

[5] Willig, A. (2008) Recent and Emerging Topics in Wireless Industrial Communications: A Selection. IEEE Transactions on Industrial Informatics, 4, 102-124. http://dx.doi.org/10.1109/TII.2008.923194

[6] Ameen, S.Y. and Nourildean, S.W. (2013) Coordinator and Router Investigation in IEEE 802.15.14 ZigBee Wireless Sensor Network. 2013 International Conference on Electrical, Communication, Computer, Power, and Control Engineering (ICECCPCE), Mosul, 17-18 December 2013, 130-134.

[7] Alliance, Z. (2008) ZigBee Specification [G/OL]. ZigBee, org.

[8] Stevanovic, D. and Vlajic, N. (2008) Performance of IEEE 802.15.4 in Wireless Sensor Networks with a Mobile Sink Implementing Various Mobility Strategies. 33rd IEEE Conference on Local Computer Networks, Montreal, 17 October 2008, 680-688.

[9] Sharma, M. (2014) Effective Data Flow in ZigBee Network Using OPNET. 2014 International Conference on Communications and Signal Processing (ICCSP), Melmaruvathur, 3-5 April 2014, 1155-1158.

[10] Dong, K. (2011) Performance and Fairness Enhancement in ZigBee Networks. Doctoral Dissertation, Delft University of Technology, Delft.

[11] Hammoodi, I.S., Stewart, B.G., Kocian, A. and McMeekin, S.G. (2009) A Comprehensive Performance Study of OPNET Modeler for ZigBee Wireless Sensor Networks. 3rd International Conference on Next Generation Mobile Applications, Services and Technologies, Cardiff, 15-18 September 2009, 357-362. http://dx.doi.org/10.1109/NGMAST.2009.12

[12] Wang, W., He, G. and Wan, J. (2011) Research on ZigBee Wireless Communication Technology. 2011 International Conference on Electrical and Control Engineering (ICECE), Yichang, 16-18 September 2011, 1245-1249. http://dx.doi.org/10.1109/ICECENG.2011.6057961 